\title{
Les enjeux identitaires de la controverse autour des expulsions de sans-papiers dans la presse française (2006-2010)
}

Lise Jacquez ${ }^{1}$

\begin{abstract}
[Résumé] Entre 2006 et 2010, plusieurs polémiques éclatent en France à propos de l'expulsion des étrangers en situation irrégulière. Les médias, en particulier la presse quotidienne, participent largement à ces polémiques. Dans cet article, nous proposons d'analyser les processus de construction identitaire qui s'opèrent dans le débat public et dans les récits médiatiques de cinq titres de presse (Le Monde, Libération, Le Figaro, Le Parisien et l'Humanité).

Mots-clés : Sans-papiers, problème public, constructions identitaires, médias, analyse de discours.

[Abstract] Between 2006 and 2010, several debates emerged in France over the deportation of migrants in irregular situation. The media, especially the daily press, largely participated in these debates. This contribution aims at analyzing the construction of identities in the debate as well as in the narratives proposed by five french newspapers (Le Monde, Libération, Le Figaro, Le Parisien et l'Humanité).

Keywords: Undocumented migrants, social problem, construction of identity, media, discourse analysis.
\end{abstract}

En 2003, sous l’impulsion du ministre de l'Intérieur de l'époque, Nicolas Sarkozy, le gouvernement français décide de fixer aux préfets des objectifs chiffrés en matière de reconduites à la frontière des étrangers en situation irrégulière. La mise en place de ces objectifs correspond à une volonté d'améliorer le taux d'exécution, traditionnellement bas $^{2}$, des décisions de reconduites à la frontière. Il s'agit également de convaincre l'opinion publique de l'efficacité de la politique de lutte contre l'immigration irrégulière et du volontarisme gouvernemental en la matière. Entre 2003 et 2010, plusieurs mesures sont prises pour atteindre les objectifs. La durée de rétention ${ }^{3}$ maximale passe de 12 à 32 jours en 2003, puis à 45 jours en 2010. De nouveaux centres de rétention sont construits et les interpellations connaissent une hausse significative ${ }^{4}$.

1 Docteure en Sciences de l'Information et de la Communication. ATER à Sciences Po Lyon. Laboratoire ELICO, Lyon.

2 Taux qui se situe autour de 22\% des mesures de reconduites prononcées annuellement dans les années 2000.

3 La rétention administrative est une mesure d'enfermement des étrangers en situation irrégulière qui est mise en œuvre le temps d'organiser le renvoi des personnes dans leur pays d'origine.

4 Elles passent d'environ 30000 par an en 1999 à 60000 en 2003 et à 111000 en 2008. 
En conséquence, le nombre d'étrangers expulsés chaque année augmente rapidement. En 2003, 11692 expulsions sont ordonnées ; 23831 en 2006. En 2008, le nombre d'expulsés s'élève à 29796 et à 32912 en $2011^{5}$. Ces mesures répressives ont suscité de nombreuses réactions dans la société française, prenant la forme de mobilisations et de dénonciations publiques de la politique gouvernementale. Les associations historiques de défense des étrangers comme la Cimade et le Gisti ${ }^{6}$ sont en première ligne de ces mobilisations. Mais l'on trouve également de nouveaux acteurs militants, comme le Réseau éducation sans frontières ${ }^{7}$, créé en 2004 pour défendre les enfants et les jeunes majeurs sans titre de séjour et scolarisés en France. À partir de 2007, la création controversée d'un ministère de l'Immigration, de l'Intégration, de l'Identité nationale et du Co-développement renforce les critiques à l'égard des expulsions. Le sujet est abondamment traité par la presse quotidienne française : entre 2006 et 2010, nous avons ainsi recensé 362 articles dans Le Monde à propos des expulsions, 733 dans Libération, 904 dans l'Humanité, 315 dans Le Parisien (édition nationale) et 288 dans Le Figaro. La lecture des articles produits par la presse quotidienne française durant cette période montre que le sujet des expulsions surgit principalement dans l'espace public sous la forme de polémiques à propos d'événements divers, tels que des cas d'expulsion individuelle dénoncés par les associations, des arrestations violentes, des mobilisations ou encore des révoltes dans les centres de rétention.

Pour Ruth Amossy et Marcel Burger «la polémique en tant qu'orchestrée par les médias contribue à la construction identitaire du corps social par la définition, la contestation et la négociation des critères qui fondent une identité citoyenne » (2011, p. 24). Dans le cas du débat sur les expulsions de sans-papiers, les enjeux identitaires concernent tout autant la question de la reconnaissance sociale voire politique des étrangers sans-papiers que les représentations de la société française et la définition d'une identité collective basée sur des valeurs partagées. Comme le résume Patrick Weil, «l'immigration (...) [peut symboliser] le danger pour l'ordre, l'unité, voire l'existence de la communauté nationale française ; ou au contraire, [représenter] les valeurs fondatrices de la communauté politique en lui donnant son universalité : les «

\footnotetext{
5 Chiffres tirés du rapport au Parlement du Secrétariat général du Comité interministériel du contrôle de l'immigration intitulé «Les chiffres de la politique d'immigration et d'intégration, Année 2011 », Décembre 2012, disponible en ligne : http://www.ladocumentationfrancaise.fr/var/storage/rapports-publics/134000159/0000.pdf (consulté le 3 janvier 2014).

6 La Cimade est une association créée en 1939 qui défend les droits des étrangers en France et intervient dans les centres de rétention. Le Gisti, créé en 1972, est un groupe d'information et de soutien aux immigrés qui mène un combat juridique pour faire respecter et élargir les droits des étrangers en France.

7 Ce réseau est issu du milieu scolaire et composé principalement d'enseignants et de parents d'élèves. II est au cœur des mobilisations de cette période.
} 
droits de l’homme », la « liberté », l’ « égalité » ou la « fraternité » (1991, p. 89). Dans cet article, nous proposons d'analyser ces enjeux et de comprendre comment les journaux français ont participé à la construction de figures spécifiques des sans-papiers ainsi qu'à la définition des frontières et des valeurs du corps social français. Notre article s'organise en trois parties. Nous commençons par préciser le cadre théorique et méthodologique de notre enquête. Dans un second temps, nous présentons les différentes manières dont la question des sans-papiers est problématisée en France. Nous terminons notre propos par une présentation des principaux résultats de l'analyse d'un corpus de presse que nous avons constitué lors de notre recherche doctorale ${ }^{8}$.

\section{Cadrage théorique et méthodologique}

Notre analyse s'inscrit dans une approche constructiviste des problèmes publics. Cette approche considère que les "problèmes publics » n'existent pas de manière autonome et objective, et qu'un phénomène social ne devient un problème public qu'à travers un processus de problématisation opéré par un certain nombre d'acteurs du champ social et politique. Il n’y a donc pas de lien mécanique entre la gravité « objective » d'un fait social et son émergence en tant que problème (Neveu, 1999, p. 42). Dans ce processus de problématisation, la dimension discursive est centrale. En effet, la problématisation d'une thématique et sa publicisation ne peuvent s'opérer que dans et par le discours, qu'il s'agisse de faire un diagnostic des enjeux, d'analyser les causes d'une situation, d'en désigner les responsables ou encore d'émettre des revendications. Dans cet article, nous nous focalisons donc sur les types de problématisation privilégiés par les acteurs du débat (principalement le gouvernement, les associations de défense des étrangers et les médias) pour formuler leurs prises de position ou leurs revendications. En nous appuyant sur une lecture de notre corpus de presse et de nombreux discours produits par le gouvernement et les associations, nous avons ainsi identifié quatre "cadres interprétatifs» dominants dans le débat sur les expulsions ${ }^{9}$. Nous les présentons ci-dessous.

8 L. Jacquez, La controverse autour des expulsions de sans-papiers dans la presse française (20062010). Analyse des discours et des enjeux sociopolitiques, Thèse de doctorat, soutenue le 8 décembre 2014, Université Lyon 2.

9 Le concept de "cadrage interprétatif » renvoie aux définitions et aux interprétations d'un problème public que construisent les acteurs sociaux (groupes mobilisés, médias, autorités) engagés dans un conflit social et/ou un débat public. Suivant une méthode assez similaire à celle préconisée par Baldwin Van Gorp (2005), nous avons sélectionné, pour chacun des cadrages, des composantes que nous avons définies et précisées (statut du migrant, cause du problème, registre normatif de justification, attribution de responsabilité, etc.). Ces composantes deviennent des indicateurs dans notre analyse du corpus de presse, et nous permet- 
Toujours, dans la mise en œuvre de cette analyse générale du débat, nous prêtons une attention particulière aux médias. En effet, l'espace médiatique, en particulier la presse écrite, a été le lieu principal de conjonction et de confrontation des discours portés par les différents acteurs engagés dans la controverse autour des expulsions. Les journaux ont contribué à la publiciser, à en construire et à en cristalliser les enjeux. L'approche adoptée ici consiste donc à analyser ces discours médiatiques comme des manifestations symboliques des logiques sociopolitiques et identitaires qui sous-tendent le problème public de l'immigration irrégulière. Nous présenterons les résultats d'une analyse menée sur un corpus composé de 2602 articles publiés par cinq quotidiens nationaux entre 2006 et 2010 : Le Monde, Libération, Le Parisien (édition nationale), l'Humanité et Le Figaro. Dans un premier temps, nous avons mené une analyse de contenu à l'aide du logiciel Modalisa, qui permet de créer une grille d'enquête dont les catégories sont définies par l'analyste. Chaque unité du corpus (pour nous, chaque article de presse) est ensuite codé par le chercheur en suivant cette grille. Les éléments codés peuvent donner lieu à un traitement statistique et comparatif. Nous avons articulé cette approche quantitative à des analyses plus qualitatives menées sur les Unes, les éditoriaux et quelques événements (mobilisation du Réseau éducation sans frontières en 2006, incendies dans les centres de rétention en 2008).

\section{Les cadres interprétatifs du débat sur les expulsions et leurs enjeux identitaires}

L'exploration des discours politiques, militants et médiatiques montre qu'il existe plusieurs manières de problématiser et, donc, de donner du sens à la présence des sans-papiers dans la société française et aux questions que cette présence soulève. Nous présentons rapidement ces différents cadrages en insistant sur les constructions identitaires spécifiques qu'ils autorisent.

\subsection{Le registre sécuritaire}

Dans une perspective sécuritaire, adoptée par les pays de l'Union européenne, les migrations sont définies comme une menace pour l'ordre public et social, ainsi que pour l'identité des sociétés d'accueil (Duez, 2008). Suivant cette logique, les migrants sont perçus soit comme des délinquants qui franchissent les frontières sans autorisation,

tent de mettre en relation les observables en discours avec les cadrages interprétatifs prédéfinis. 
soit comme une foule indistincte de "pauvres». À ce titre, la lutte contre l'immigration clandestine est une question politiquement consensuelle en Europe et en France. Les stratégies de contrôle des frontières européennes visent ainsi à protéger les espaces nationaux d'un « en-dehors » perçu comme une source d'insécurité.

\subsection{Ouverture des frontières et liberté de circulation}

Le discours sur l'ouverture des frontières s'oppose point par point à cette vision sécuritaire de l'immigration, et défend au contraire le principe de la liberté de circulation. En France, ce combat politique est principalement porté par des acteurs associatifs comme le Gisti, ainsi que par des chercheurs et par certains organismes de l'ONU. Le principe de la liberté de circulation se base sur la reconnaissance d'un monde ouvert et interdépendant dans lequel la mobilité devrait être considérée comme un fait sociologique normal et structurel ${ }^{10}$. D'un point de vue politique et juridique, l'objectif de cette approche est de faire des migrants des sujets de droit à part entière. Pour cela, la reconnaissance d'un droit à la mobilité et à l'installation dans un pays autre que le sien est fondamentale. Mais, pour être réalisée, cette normalisation des migrations doit s'accompagner d'une transformation des manières de penser et de représenter la société française, afin de promouvoir une société ouverte sur le reste du monde plutôt que repliée sur elle-même. Cette conception des flux migratoires est très radicale au regard de l'état du débat actuel en France, dans lequel domine une conception sécuritaire de l'immigration. Pour cette raison, d'autres modes de critiques des expulsions et de défense des sans-papiers, moins subversifs, existent. Ils sont également portés par des associations et des collectifs de défense des sans-papiers.

\subsection{Le registre de l'intégration}

Le recours à l'argument de l' "intégration » est très courant dans les plaidoyers militants contre les expulsions. La longévité du séjour en France, la possession d'un travail et l'existence d'une vie familiale et sociale sont les trois critères principaux qui fondent généralement cet argument. Dans les années 2000, le Réseau Education sans Frontières (RESF), créé pour défendre les familles sans-papiers ayant des enfants scolarisés en France et menacées d'expulsion, base ainsi une bonne partie de son argumentaire sur l'intégration des familles. Il s'agit d'opposer une réalité vécue l'intégration sociale et professionnelle - à une exclusion juridique décidée par le système étatique. À travers la mobilisation de citoyens qui soutiennent les sans-papiers,

10 B. Badie et al., Pour un autre regard sur les migrations. Construire une gouvernance mondiale, La Découverte, 2008. 
ce registre permet également de produire l'image d'une société solidaire, guidée par des valeurs d'égalité et d'hospitalité, valeurs qui apparaissent comme menacées par la politique migratoire. Cependant, l'argument de l'intégration ne permet pas toujours de revendiquer efficacement des droits de portée générale, car il présuppose une démonstration de l'intégration concrète des personnes qui peut facilement déboucher sur une individualisation du problème. Plaider la cause de chaque migrant ou famille de migrants est alors à la fois ce qui assure le succès de la revendication et ce qui la dépolitise car ce mode de défense prend le risque d'assimiler la notion d' " intégration » à l'idée de mérite individuel. Cette idée de mérite individuel est d'ailleurs privilégiée par les pouvoirs publics ${ }^{11}$. Enfin, soulignons que l'argument de l'intégration ne vise que les migrants déjà installés dans la société, limitant ainsi la question des migrations à un enjeu interne à l'espace national.

\subsection{Refus de la répression et défense des valeurs républicaines}

Ce dernier cadrage est spécifique au débat sur les expulsions ${ }^{12}$. Il renvoie aux discours militants et médiatiques qui problématisent l'action répressive de l'État en termes d'atteinte aux valeurs et aux principes fondamentaux de la République française. La mise en place de quotas d'expulsions a, en effet, provoqué une augmentation très importante des moyens mis en œuvre pour les atteindre. Dans ce contexte, la répression policière devient plus visible et, surtout, perd son caractère exceptionnel pour devenir routinière : les mesures répressives sont, en effet, présentées comme la réponse politique principale à l'immigration irrégulière. La question soulevée par les protestataires est celle des limites et des justifications du recours à la violence étatique vis-àvis des étrangers, dans une société démocratique. Dans cette perspective, ce n'est pas nécessairement la prérogative étatique en matière de refus de séjour et d'éloignement du territoire qui est mise en cause, mais plutôt le fait que cette prérogative soit instrumentalisée politiquement et, donc, que les moyens répressifs mis en œuvre soient déconnectés de la poursuite d'une fin raisonnable. Aux yeux des protestataires, élever la répression des personnes en situation irrégulière au rang d'enjeu politique de pre-

11 C'est ce dont témoignent les critères de régularisation fixés par les circulaires de 2006 (prise par Nicolas Sarkozy) et de 2012 (prise par Manuel Valls) : à côté de critères objectifs comme la durée de séjour ou la scolarisation, d'autres critères induisent l'idée de mérite et ouvrent un espace d'évaluation arbitraire aux préfectures: parcours scolaire sérieux et assidu, réelle volonté d'intégration, etc.

12 Par opposition aux deux premiers cadrages analysés qui sont mobilisés plus largement pour problématiser la situation des sans-papiers en France, même en dehors d'événements mettant en scène l'action répressive de l'État. 
mier ordre, pourrait alors bien faire ressurgir le spectre d'un État autoritaire et policier bafouant les droits fondamentaux des étrangers. Les allusions au régime de Vichy ou aux années 1930, ont, en effet, été fréquentes de la part de différents défenseurs des sans-papiers lors de la période étudiée. Certaines polémiques liées à la politique de reconduites à la frontière ne résultent donc pas d'une prise de position de fond sur la situation des étrangers en situation irrégulière et sur les lois régissant le droit au séjour, mais découlent plus directement du refus d'une violence étatique trop explicite à l'encontre des étrangers.

\section{Le rôle des médias}

Les manières de problématiser les expulsions de sans-papiers sont donc variées et ne visent pas toutes les mêmes objectifs politiques et symboliques. Par ailleurs, ces différents registres ne bénéficient pas tous du même écho médiatique durant la période étudiée (2006-2010). Ainsi la revendication d'une ouverture des frontières apparait difficilement audible dans l'espace médiatique tant elle nécessite une redéfinition complète des termes du débat, opération couteuse en temps et complexe en raisonnement, qui ne s'accorde, a priori, pas avec les modalités d'une prise de parole médiatique (brièveté et clarté du message, réaction « à chaud » à l'égard de l'événement traité). De plus, il faut noter que, dans les années 2000, le discours sur la liberté de circulation est porté par un nombre restreint d'acteurs militants, qui ne sont pas nécessairement les plus visibles sur le terrain et dans les médias. La présentation des résultats de l'analyse du corpus vont maintenant nous permettre de préciser les registres les plus mobilisés par les journaux pour rendre compte de la polémique, ainsi que d'interroger l'autonomie ou, au contraire, la dépendance des discours médiatiques vis-à-vis des discours des autres acteurs.

\subsection{Des expulsions très controversées dans la presse}

Il existe une forte visibilité médiatique de la question des expulsions de 2006 à 2010. En effet, un nombre important d'articles est publié par les différents journaux pris en compte pour la présente analyse (2602). Deux titres, Libération et l'Humanité alimentent davantage la couverture médiatique (entre $50 \%$ et $70 \%$ suivant les années) que les autres. On peut également noter, dans tous les journaux pris en compte, une évolution des sujets traités d'une année à l'autre. En 2006, en 2009 et en 2010, le débat concerne des catégories particulières de migrants (les familles, puis les migrants de Calais, puis les Roms européens), tandis qu'en 2007 et 2008, c'est la légitimité même de la politique d'éloignement et ses conséquences sur l'ensemble des sans-papiers qui est débattue. Certains journaux semblent donc relayer des discours qui participent à la production d'un dissensus sur cette politique: c'est le cas de Libération et de l'Humanité dès 2006, et on peut donc les désigner comme des précurseurs dans la 
mise en débat de cette question. C'est également le cas du Monde et du Parisien à partir de 2008. L'accroissement de la répression vis-à-vis des sans-papiers est ainsi loin de faire consensus et est, au contraire, très controversée dans la presse qui prend ses distances vis-à-vis de la communication gouvernementale.

La critique médiatique comporte deux volets : en premier lieu, ce sont les souffrances infligées aux sans-papiers qui sont mises en lumière. La mobilisation du RESF et les révoltes en rétention ${ }^{13}$ sont des occasions, pour les quotidiens, d'insister sur la détresse des personnes menacées d'expulsion en mettant en scène des figures particulièrement vulnérables (en particulier les enfants). Ensuite, trois journaux, Le Monde, l'Humanité et Libération dénoncent l'atteinte que porte cette politique à des valeurs et des principes fondamentaux de la République française (droits de l'homme, respect des étrangers, refus de la xénophobie. Entre 2006 et 2010, Libération publie, par exemple, plusieurs éditoriaux et Unes fortement dénonciateurs de la politique du gouvernement. On y trouve des titres tels que : «Arrêtez la chasse aux familles ! » ${ }^{14}$, « Déshonneur », «Honte $»^{15}$. Le 11 août 2007, le directeur de la rédaction de Libération, Laurent Joffrin condamne ainsi les arrestations à domicile en déclarant : « cette pratique [les arrestations à domicile] ne fait pas honneur à la démocratie française », et il ajoute « la France que nous voyons aujourd'hui n'est plus tout à fait, sur ce plan-là, celle que nous aimons ». On retrouve des critiques similaires dans l'Humanité qui dénonce, par exemple, "la détresse infligée aux sans-papiers vivant sur notre sol » comme un " affront aux principes fondamentaux d'une République comme la nôtre.. ${ }^{16} \mathrm{Ce}$ cadrage est le plus mobilisé dans les discours du Monde, de Libération et de l'Humanité entre 2006 et 2008.

Le registre de l'intégration est également très souvent repérable dans les discours de presse. Les journaux se focalisent sur les cas d'expulsions individuelles, privilégiant les cas de sans-papiers issus de familles socialement intégrées. En 2006, la mobilisation de nombreux enseignants et parents d'élèves contre les expulsions de familles est ainsi l'occasion, pour les quotidiens, de proposer des représentations valorisantes de la société française (lien social solide, solidarité). Tous les journaux ont ainsi illustré leurs reportages par des cas de familles présentées comme particulièrement «intégrées » et « méritantes».

13 À partir de décembre 2007 et durant toute l'année 2008, plusieurs mouvements de protestation allant de la grève de la faim à des départs d'incendie se déroulent dans plusieurs centres de rétention de la région parisienne. Plusieurs sans-papiers retenus entendent ainsi protester contre leur mise en rétention et dénoncer les quotas d'expulsion.

14 Une de Libération du 11 août 2007.

15 Titres des éditoriaux du 30 octobre et du 18 décembre 2008.

16 L'Humanité, éditorial du 22 avril 2006. 


\subsection{Un débat politique absent}

Mais, malgré ce discours dénonciateur, les journaux semblent réticents à s'affranchir de la perspective sécuritaire. Dans Libération et Le Monde, l'on trouve ainsi des formules de concession telles que celles-ci : « Il ne s'agit pas de déclarer tout de go que les frontières ont disparu (...) $\|^{17}$, "Certes, personne ne suggère d'ouvrir les frontières à tous les flux migratoires ${ }^{18}$, "Pas d'angélisme facile : aucun gouvernement responsable ne peut rester sans rien faire devant le flux de l'immigration illégale ${ }^{19}$. La médiatisation accrue des sans-papiers, dans une période de durcissement de la politique d'immigration, génère donc un régime de visibilité paradoxal : les sans-papiers sont plus visibles, la répression qui les vise est souvent dénoncée sans qu'il soit pour autant plus facile de les défendre politiquement ou de prendre position pour une autre gestion des flux migratoires. Comme le souligne le politiste Guillaume Garcia, la politisation d'un problème dépend de sa prise en charge «par des acteurs suffisamment influents pour le constituer en objet de débat sur la scène politique institutionnelle ", et il ajoute: "le problème doit alors être transformé en enjeu structuré par les clivages entre les grandes formations politiques » (Garcia, 2013, p. 208). Or, en France, il existe un consensus entre les deux grands partis, l'UMP et le Parti socialiste, sur la nécessité d'un contrôle ferme des flux migratoires. C'est pour cette raison, nous semble-t-il, que les luttes et les revendications défensives, parce qu'elles apparaissent plus « réalistes », c'est-à-dire audibles par l'État, bénéficient d'une bien meilleure couverture médiatique que les propositions politiques offensives, telle que l'ouverture des frontières.

\subsection{Un débat « nationalo-centré »}

Enfin, si un débat a bien lieu au sujet des expulsions dans la presse, il ne dépasse pas les frontières nationales. De manière très frappante, nous ne trouvons par exemple que très peu d'articles consacrés à la position des pays d'origine des migrants expulsés ou menacés de l'être, vis-à-vis de la politique française. Ainsi le "pays d'origine », qu'il soit représenté par l’État ou par la société civile, n’est mentionné comme source de critique de la politique française que dans 40 articles soit 1,4\% du corpus. En outre, seuls une cinquantaine d'articles sur les 2602 dénombrés traitent de la situation des sans-papiers après leur expulsion du territoire français. Lorsqu'il s'agit des expulsions, les migrations ne sont donc pas problématisées comme un enjeu international. Selon nous, le caractère «nationalo-centré » du débat, tel qu'il est mis en scène dans

17 Libération, 11 août 2007.

18 Libération, 24 juin 2008.

19 Le Monde, 24 juin 2008. 
les journaux, joue un rôle important dans la construction de la controverse autour des expulsions. Il conduit notamment à accorder une place écrasante aux enjeux identitaires qui traversent la société française, enjeux qui concernent à la fois les valeurs de la société, les représentations du corps social et la place accordée aux étrangers en son sein. À l'inverse, les enjeux de gouvernance internationale des migrations, qui concernent à la fois les droits sociaux, économiques et juridiques des migrants et la coopération entre pays d'origine et pays d'accueil pour mieux appréhender et gérer les flux, sont évacués du débat sur les expulsions.

\section{Conclusion}

Pour conclure, les journaux français ont bien participé à ouvrir un débat sur la légitimité de la politique chiffrée d'expulsions, mais sans soutenir d'alternatives politiques ni participer à la construction d'un nouveau regard sur les migrations. D'un côté, les quotidiens étudiés ont joué un rôle dans la construction d'un débat démocratique, en donnant de la visibilité aux différents discours s'exprimant dans la société. Ils ont donc fait preuve d'autonomie vis-à-vis du discours de l'État. Certains récits médiatiques participent également à l'intégration symbolique des sans-papiers dans la cité. De cette manière, ils participent potentiellement à la redéfinition des normes d'exclusion ou d'inclusion qui régissent l'espace national. Néanmoins, ce ne sont pas les discours d'opposition aux expulsions les plus radicaux politiquement qui ont été rendus visibles, mais des arguments plus modérés comme l'intégration de certains sans-papiers et le refus d'une répression étatique jugée démesurée. Finalement, les discours médiatiques apparaissent donc fortement dépendants de l'état du débat politique sur l'immigration.

\section{Bibliographie}

AMOSSY R., BURGER M. (2011) : «Introduction : la polémique médiatisée », Semen, nº 31.

Badie B., Devin G., Brauman R., Decaux E., Wihtol De Wenden C. (2008) : Pour un autre regard sur les migrations. Construire une gouvernance mondiale, Paris: La Découverte.

Brown W. (2009): Murs. Les murs de séparation et le déclin de la souveraineté étatique, trad. de l'anglais par N. Vieillescazes, Paris : Les Prairies ordinaires.

DUEZ D. (2008), L'Union européenne et l'immigration clandestine. De la sécurité intérieure à la construction de la communauté politique, Bruxelles: Éd. Université de Bruxelles.

GARCIA G. (2013), La cause des « sans ». Sans-papiers, sans-logis, sans-emploi à l'épreuve des médias, Rennes : Presses universitaires de Rennes. 
NEVEU E. (1999) : "L'approche constructiviste des problèmes publics. Un aperçu des travaux anglo-saxons ». In Études de communication, $n^{\circ} 22$, p. 41-58.

VAN GORP B. (2005) : «Where is the frame ? Victims and intruders in the belgium press coverage of the asylum issue ». In European Journal of Communication, $\mathrm{n}^{\circ} 20$, pp. 484-507.

WEIL P. (1991), La France et ses étrangers. L'aventure d'une politique d'immigration, 19381991, Paris : Éditions Calman-Lévy. 